PROCEEDINGS OF THE

AMERICAN MATHEMATICAL SOCIETY

Volume 128, Number 11, Pages 3435-3444

S 0002-9939(00)05392-2

Article electronically published on May 2, 2000

\title{
DIFFERENTIABLE MAPPINGS WITH AN INFINITE NUMBER OF CRITICAL POINTS
}

\author{
C. PINTEA
}

(Communicated by Ralph Cohen)

\begin{abstract}
In this paper we shall give some sufficient conditions in order that
\end{abstract} the so-called $\varphi$-category of a pair $(M, N)$ of differentiable manifolds be infinite.

\section{INTRODUCTION}

Let $M, N$ be compact connected differentiable (smooth) manifolds having the same dimension $m \geq 2$. If $\pi_{q}(M) \nsucceq \pi_{q}(N)$ for some $q \geq 2$, then any differentiable (smooth) mapping $f: M \rightarrow N$ has one critical point at least. Indeed, otherwise $f$ is a covering map and $\pi_{q}(M) \cong \pi_{q}(N)$, for $q \geq 2$, follows easily.

This observation justifies the investigations on the cardinal number

$$
\varphi(M, N)=\min \left\{|C(f)| \mid f \in C^{\infty}(M, N)\right\}
$$

called the $\varphi$-category of the pair $(M, N)$, where $M, N$ are, at this time, arbitrary differentiable manifolds and $C(f)$ is the critical set of $f \in C^{\infty}(M, N)$. If any differentiable mapping from $M$ to $N$ has infinitely many critical points, we shall use the notation $\varphi(M, N)=\infty$. As we have already mentioned in our previous papers $\mathrm{AnPi}$ and [Pi1], $\varphi(M, N)$ represents a measure of non-immersability of $M$ into $N$, if $\operatorname{dim} M<\operatorname{dim} N$ and it is a measure of the distance of the pair $(M, N)$ from a fibration if $\operatorname{dim} M \geq \operatorname{dim} N$ and $M, N$ are compact differentiable manifolds. Some results concerning the $\varphi$-category of the pair $(M, \mathbf{R})$ are obtained in GoGo] and [Ta]. For the equivariant (invariant) situation see also [Da].

The main result of this paper is Theorem 3.1 which asserts that, for two given compact connected differentiable manifolds $M$ and $N$ having the same dimension $m, \varphi(M, N)$ is infinite in the following conditions:

(i) $m \geq 3$ and $\pi_{1}(N)$ has no subgroup isomorphic with $\pi_{1}(M)$;

(ii) $m \geq 4$ and $\pi_{q}(M) \neq \pi_{q}(N)$ for some $q \in\{2,3, \ldots, m-2\}$.

The proof of Theorem 3.1 is essentially based on the technical Theorems 1.1, 1.2 and on Proposition 2.3 and it is very easy to cover. Proposition 2.3 is an immediate consequence of Theorem 2.2 and the last one could be also considered an important result in itself. Theorem 2.2 roughly asserts that for two given manifolds $P$ and $M$ such that $\operatorname{dim} P<\operatorname{dim} M, \partial P \neq \emptyset, \partial M=\emptyset$ and a discrete subset $A$ of $M$ whose derived set $A^{\prime}$ (i.e. the set of accumulation points) is also discrete, in each

Received by the editors October 16, 1997 and, in revised form, December 18, 1998.

2000 Mathematics Subject Classification. Primary 55Q05, 57R70, 57S25.

Key words and phrases. G-manifolds, critical points, critical orbits, homotopy groups.

This paper is a part of the author's doctoral dissertation. 
homotopy class $[f] \in[(P, \partial P),(M, M \backslash \bar{A})]$ there is a map whose image avoids the set $\bar{A}$. For its proof $f$ is assumed to be smooth and around the common points of the sets $\bar{A}$ and $\operatorname{Im} f$ we take small balls such that they don't meet $\bar{A}$ again. Using Sard's theorem we can consider in each such ball a point which doesn't belong to $\operatorname{Im} f$. Further on, project the common points of $\operatorname{Im} f$ and a given ball on the border of that ball along some certain curves starting from the considered point of the ball and passing through their common points. The asserted mapping in the class $[f]$ is obtained by composing those projections with the initial mapping $f$.

\section{Preliminary Results}

In their paper $[\mathrm{FaHu}$, E. Fadell and S. Husseini proved, using the standard technique of Ljusternik-Schnirelmann category, that a $C^{1}$-functional $f: \Lambda \rightarrow \mathbf{R}$ has an unbounded sequence of critical values (therefore infinitely many critical points), under certain hypothesis on the open subset $\Lambda$ of the Hilbert space $E$ and on the functional $f$. Our first result proves a similar fact for a mapping between two connected differentiable manifolds.

As we have already defined before, $C(f)$ denotes the critical set of the mapping $f: M \rightarrow N$. Recall that $R(f)$ denotes the regular set of $f$ and $B(f)=f(C(f))$ denotes the set of critical values of $f$.

Theorem 1.1. Let $M, N$ be connected differentiable manifolds such that $\operatorname{dim} M \geq$ $\operatorname{dim} N \geq 2$. If $f: M \rightarrow N$ is a non-surjective closed differentiable mapping, then either $C(f)=M$, or $f$ has infinitely many critical values. Therefore $f$ has infinitely many critical points. If $M$ is compact and $N$ is noncompact, then one particularly gets that $\varphi(M, N)=\infty$.

Proof. Let us first prove that $f^{-1}(\partial \operatorname{Im} f) \subseteq C(f)$, which implies that $\partial \operatorname{Im} f \subseteq$ $B(f)$. Indeed, otherwise $f^{-1}(\partial \operatorname{Im} f) \cap R(f) \neq \emptyset$ and $f$ is locally open around any point of the set $f^{-1}(\partial \operatorname{Im} f) \cap R(f)$. If $x \in f^{-1}(\partial \operatorname{Im} f) \cap R(f)$ is a fixed point and $U$ is an open neighbourhood of $x$ such that $\left.f\right|_{U}: U \rightarrow N$ is open, then $f(U)$ is particularly open. But this is a contradiction to the fact that $f(x) \in \partial \operatorname{Im} f$.

If $C(f) \neq M$, it follows, by Sard's theorem, that $\operatorname{Im} f \backslash B(f) \neq \emptyset$. In the following we shall show that $N \backslash B(f)$ is not connected, which means that $B(f)$ is infinite. Indeed, if $y \in \operatorname{Im} f \backslash B(f), y^{\prime} \in N \backslash \operatorname{Im} f$, then obviously $y, y^{\prime} \in N \backslash B(f)$. Consider $\gamma:[0,1] \rightarrow N$ a continuous path joining $y$ to $y^{\prime}$. Because $y \in \operatorname{Im} f$ and $y^{\prime} \in$ $N \backslash \operatorname{Im} f$, it follows that $\gamma([0,1])$ intersects the border $\partial \operatorname{Im} f$ and hence the set $B(f)$.

Theorem 1.2. Let $M, N$ be compact connected differentiable manifolds having the same dimension $m \geq 2$. If the differentiable mapping $f: M \rightarrow N$ is surjective and has finitely many critical points, then the set $f^{-1}(B(f))$ is the closure of a discrete set whose derived set is finite, and the restriction

$$
\left.f\right|_{M \backslash f^{-1}(B(f))}: M \backslash f^{-1}(B(f)) \rightarrow N \backslash B(f)
$$

is a covering mapping with finitely many sheets.

Proof. Before proving the required statement we shall prove the inclusion

$$
\left[f^{-1}(B(f)) \cap R(f)\right]^{\prime} \subseteq C(f)
$$

where $\left[f^{-1}(B(f)) \cap R(f)\right]^{\prime}$ is the derived set of $\left[f^{-1}(B(f)) \cap R(f)\right]$. Indeed, if the above inclusion is not true, then there exists a critical value $y \in B(f)$ whose fiber 
$f^{-1}(y)$ has a regular point $x$ as accumulation point. But this is a contradiction to the fact that $f$ is a local diffeomorphism around of $x$. Further on, we have successively:

$$
\begin{gathered}
f^{-1}(B(f))=\overline{f^{-1}(B(f)) \cap M}=\overline{f^{-1}(B(f)) \cap[C(f) \cup R(f)]} \\
=\overline{\left(f^{-1}(B(f)) \cap C(f)\right) \cup\left[f^{-1}(B(f)) \cap R(f)\right]}=\overline{C(f) \cup\left[f^{-1}(B(f)) \cap R(f)\right]} .
\end{gathered}
$$

Obviously

$$
C(f) \cup\left[f^{-1}(B(f)) \cap R(f)\right]
$$

is a discrete set whose derived set $\left[f^{-1}(B(f)) \cap R(f)\right]^{\prime}$ is finite, being a subset of $C(f)$, and $\left.f\right|_{M \backslash f^{-1}(B(f))}$ is a local diffeomorphism. Therefore to prove what is required, it is enough to show that $\left|f^{-1}(y)\right|=\left|f^{-1}\left(y^{\prime}\right)\right|<\infty$ for all $y, y^{\prime} \in N \backslash B(f)$. Let us first see that $f^{-1}(y)$ is finite for all $y \in N \backslash B(f)$. Indeed, otherwise $f^{-1}(y)$ has accumulation points obviously contained in $f^{-1}(y)$. But this contradicts the fact that $f$ is locally a diffeomorphism around any point $x \in f^{-1}(y)$. It remains to prove that the number $\left|f^{-1}(y)\right|$ is invariant when $y$ runs over the set $N \backslash B(f)$. We first prove this statement locally, that is, every $y \in N \backslash B(f)$ has an open neighbourhood $V_{y}$ such that $\left|f^{-1}(y)\right|=\left|f^{-1}\left(y^{\prime}\right)\right|<\infty$ for all $y^{\prime} \in V_{y}$. Assume that $f^{-1}(y)=\left\{x_{1}, \ldots, x_{p}\right\}$. Consider $U_{1}, U_{2}, \ldots, U_{p}$ open neighbourhoods of $x_{1}, x_{2}, \ldots, x_{p}$ respectively, and $V$ an open neighbourhood of $y$ such that $f\left(U_{i}\right)=V$ and $\left.f\right|_{U_{i}}: U_{i} \rightarrow V$ is a diffeomorphism. Hence $\left|f^{-1}(y)\right| \leq\left|f^{-1}\left(y^{\prime}\right)\right|$, for all $y^{\prime} \in V$, and assuming that there exists a sequence $\left\{y_{n}\right\}_{n \in \mathbf{N}} \subseteq V$ such that $y_{n} \rightarrow y$ and $\left|f^{-1}\left(y_{n}\right)\right|>\left|f^{-1}(y)\right|$, it follows that one can find $x_{n} \in M \backslash\left(\bigcup_{i=1}^{p} U_{i}\right)$ such that $f\left(x_{n}\right)=y_{n}$. Because $M$ is compact, $\left\{x_{n}\right\}_{n \in \mathbf{N}}$ has a convergent subsequence denoted in the same way, that is, there exists $x_{0}=\lim _{n \rightarrow \infty} x_{n}$. But since $x_{n} \in M \backslash\left(\bigcup_{i=1}^{p} U_{i}\right)$ for all $n \in \mathbf{N}$, it follows on one hand that $x_{0} \in M \backslash\left(\bigcup_{i=1}^{p} U_{i}\right)$, and on the other hand, passing to the limit in the equality $f\left(x_{n}\right)=y_{n}$, that $f\left(x_{0}\right)=y$. This means that $x_{0} \in\left\{x_{1}, x_{2}, \ldots, x_{p}\right\} \subseteq \bigcup_{i=1}^{p} U_{i}$. Therefore there exists an open neighbourhood $V_{y} \subset V$ such that $\left|f^{-1}(y)\right|=\left|f^{-1}\left(y^{\prime}\right)\right|$ for all $y^{\prime} \in V_{y}$.

Further on, take two arbitrary points $y, y^{\prime} \in N \backslash B(f)$ and consider a continuous path $\gamma:[0,1] \rightarrow N \backslash B(f)$ joining $y$ to $y^{\prime}$, i.e. $\gamma(0)=y, \gamma(1)=y^{\prime}(N \backslash B(f)$ is connected, $B(f)$ being a finite set), and cover $\gamma([0,1])$ with a finite number of above constructed open sets by means of which one can easily prove that $\left|f^{-1}(y)\right|=$ $\left|f^{-1}\left(y^{\prime}\right)\right|$, and the theorem is completly proved.

\section{Some homotopical ASPECTS}

For $r>0$ and $n \in \mathbf{N}^{*}$ denote by $D_{r}^{n}$ and $S_{r}^{n-1}$ the open disk and the sphere respectively, having the center at the origin of the space $\mathbf{R}^{n}$ and radius $r . D_{1}^{n}$ and $S_{1}^{n-1}$ will be simply denoted by $D^{n}$ and $S^{n-1}$ respectively. by

For $x_{0} \in D^{n}$, define the continuous mapping $h_{x_{0}}: \mathbf{R}^{n} \backslash\left\{x_{0}\right\} \rightarrow \mathbf{R}^{n} \backslash\left\{x_{0}\right\}$ given

$$
h_{x_{0}}(x)= \begin{cases}x & \text { if } x \in \mathbf{R}^{n} \backslash D^{n} \\ x_{0}+\alpha(x)\left(x-x_{0}\right) & \text { if } x \in \bar{D}^{n}\end{cases}
$$

where

$$
\alpha(x)=\left\langle\frac{x_{0}}{\left\|x-x_{0}\right\|}, \frac{x_{0}-x}{\left\|x-x_{0}\right\|}\right\rangle+\sqrt{\left\langle\frac{x_{0}}{\left\|x-x_{0}\right\|}, \frac{x_{0}-x}{\left\|x-x_{0}\right\|}\right\rangle^{2}+\frac{1-\left\|x_{0}\right\|^{2}}{\left\|x-x_{0}\right\|^{2}}} .
$$




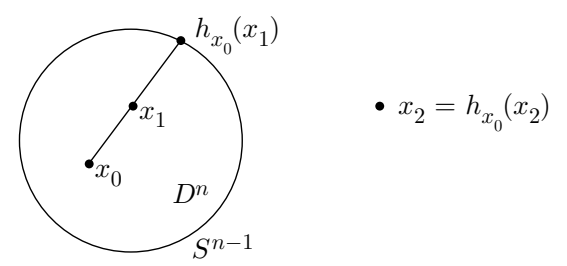

FiguRe 1.

The mapping $h_{x_{0}}$ acts on $\mathbf{R}^{n} \backslash\left\{x_{0}\right\}$ as in Figure 1,

Let $M$ be an $n$-dimensional manifold and $c=(U, \varphi)$ be a local chart of $M$ such that $\bar{D}^{n} \subseteq \varphi(U)$. Denote by $D_{\varphi}$ and by $S_{\varphi}$ the sets $\varphi^{-1}\left(D^{n}\right)$ and $\varphi^{-1}\left(S^{n-1}\right)$ respectively. For $x_{0} \in D_{\varphi}$ define the continuous mapping $h_{c, x_{0}}: M \backslash\left\{x_{0}\right\} \rightarrow M \backslash\left\{x_{0}\right\}$ given by

$$
h_{c, x_{0}}(x)= \begin{cases}x & \text { if } x \in M \backslash D_{\varphi}, \\ \left(\left.\varphi^{-1} \circ h_{\varphi\left(x_{0}\right)}\right|_{\varphi(U)} \circ \varphi\right)(x) & \text { if } x \in U .\end{cases}
$$

Remarks. (i) $h_{x_{0}}\left(D^{n} \backslash\left\{x_{0}\right\}\right)=S^{n-1}$ and $\left.h_{x_{0}}\right|_{S^{n-1}}=i d_{S^{n-1}}$.

(ii) $h_{x_{0}} \simeq_{H^{x_{0}}} i d_{\mathbf{R}^{n} \backslash\left\{x_{0}\right\}}\left(\right.$ rel $\left.\mathbf{R}^{n} \backslash D^{n}\right)$, where $H^{x_{0}}:\left(\mathbf{R}^{n} \backslash\left\{x_{0}\right\}\right) \times[0,1] \rightarrow \mathbf{R}^{n} \backslash\left\{x_{0}\right\}$ is given by $H^{x_{0}}(x, t)=(1-t) x+t h_{x_{0}}(x)$.

(iii) $h_{c, x_{0}}\left(D_{\varphi} \backslash\left\{x_{0}\right\}\right)=S_{\varphi}$ and $\left.h_{c, x_{0}}\right|_{S_{\varphi}}=i d_{S_{\varphi}}$.

(iv) Let $c_{1}=\left(U_{1}, \varphi_{1}\right), \ldots, c_{k}=\left(U_{k}, \varphi_{k}\right)$ be local charts in $M$ such that for $i \neq j$ $U_{i} \cap U_{j}=\emptyset$ and $D^{n} \subseteq \bigcap_{i=1}^{k} \varphi_{i}\left(U_{i}\right)$. If $x_{i} \in D_{\varphi_{i}}, i \in\{1,2, \ldots, k\}$, then

$$
g_{c_{1}, x_{1}} \circ \ldots \circ g_{c_{k}, x_{k}}=g_{c_{\pi(1)}, x_{\pi(1)}} \circ \ldots \circ g_{c_{\pi(k)}, x_{\pi(k)}} \text {, }
$$

where $\pi$ is an element of the symmetric group of the set $\{1,2, \ldots, k\}$ and

$$
g_{c_{i}, x_{i}}: M \backslash\left\{x_{1}, \ldots, x_{k}\right\} \rightarrow M \backslash\left\{x_{1}, \ldots, x_{k}\right\}
$$

are given by $g_{c_{i}, x_{i}}=\left.h_{c_{i}, x_{i}}\right|_{M \backslash\left\{x_{1}, \ldots, x_{k}\right\}}$, for all $i \in\{1,2, \ldots, k\}$.

(v) Under the conditions of the statement (iv), it is also true that

$$
g_{c_{1}, x_{1}} \circ \ldots \circ g_{c_{k}, x_{k}} \simeq_{G_{c_{1} \ldots c_{k}}^{x_{1} \ldots x_{k}}} i d_{M \backslash\left\{x_{1}, \ldots, x_{k}\right\}}\left(\operatorname{rel} M \backslash \bigcup_{i=1}^{k} D_{\varphi_{i}}\right)
$$

where $G_{c_{1} \cdots c_{k}}^{x_{1} \cdots x_{k}}:\left(M \backslash\left\{x_{1}, \ldots, x_{k}\right\}\right) \times[0,1] \rightarrow M \backslash\left\{x_{1}, \ldots, x_{k}\right\}$ is given by

$$
G_{c_{1} \cdots c_{k}}^{x_{1} \cdots x_{k}}(x, t)= \begin{cases}x & \text { if } x \in M \backslash \bigcup_{i=1}^{k} D_{\varphi_{i}}, \\ \varphi_{1}^{-1}\left(H^{\varphi_{1}\left(x_{1}\right)}\left(\varphi_{1}(x), t\right)\right) & \text { if } x \in \bar{D}_{\varphi_{1}}, \\ \vdots & \\ \varphi_{k}^{-1}\left(H^{\varphi_{k}\left(x_{k}\right)}\left(\varphi_{k}(x), t\right)\right) & \text { if } x \in \bar{D}_{\varphi_{k}} .\end{cases}
$$

Lemma 2.1. Let $M_{1}$ be a differentiable manifold with boundary, $M_{2}$ be a differentiable manifold without boundary, $C$ be a closed subset of $M_{2}$ and $f: M_{1} \rightarrow M_{2}$ be a continuous mapping such that $f\left(\partial M_{1}\right) \subseteq M_{2} \backslash C$. Then there exists a homotopy $H: M_{1} \times[0,1] \rightarrow M_{2}$ such that $f=H(\cdot, 0), H_{t}\left(\partial M_{1}\right) \subseteq M_{2} \backslash C$ for all $t \in[0,1]$ and $H(\cdot, 1)$ is a differentiable mapping. 
Proof. There exists a homotopy $K: \partial M_{1} \times[0,1] \rightarrow M_{2} \backslash C$ such that $K(\cdot, 0)=\left.f\right|_{\partial M_{1}}$ and $K(\cdot, 1)$ is a differentiable mapping (see [Go Proposition 4.6, pp. 64]). Consider the homotopy $H^{2}: M_{1} \times[0,1] \rightarrow M_{2}$ :

$$
H^{2}(x, t)= \begin{cases}K\left(\left(\pi_{1} \circ Q^{-1}\right)(x), 1\right) & \text { if } x \in Q\left(\partial M_{1} \times[0,1-t]\right), \\ K\left(\left(\pi_{1} \circ Q^{-1}\right)(x), \frac{2-\left(\pi_{2} \circ Q^{-1}\right)(x)}{t+1}\right) & \text { if } x \in Q\left(\partial M_{1} \times[1-t, 2]\right), \\ f\left(Q\left(\left(\pi_{1} \circ Q^{-1}\right)(x), 3\left(\pi_{2} \circ Q^{-1}\right)(x)-6\right)\right) & \text { if } x \in Q\left(\partial M_{1} \times[2,3]\right), \\ f(x), & \text { if } x \in M_{1} \backslash Q\left(\partial M_{1} \times[0,3)\right),\end{cases}
$$

where $Q: \partial M_{1} \times[0, \infty) \rightarrow U \subseteq M_{1}$ is a collar neighbourhood of the boundary $\partial M_{1}$ ( $U$ is an open neighbourhood of the boundary $\partial M_{1}$ and $Q$ is a diffeomorphism such that $\left.Q(x, 0)=x, \forall x \in \partial M_{1}\right)$ and $\pi_{1}: \partial M_{1} \times[0, \infty) \rightarrow \partial M_{1}, \pi_{2}: \partial M_{1} \times[0, \infty) \rightarrow$ $[0, \infty)$ are the projections.

Consider the homotopy $H^{3}: M_{1} \times[0,1] \rightarrow M_{2}$ :

$$
H^{3}(x, t)= \begin{cases}K\left(\left(\pi_{1} \circ Q^{-1}\right)(x), 1-t-\frac{1}{2}\left(\pi_{2} \circ Q^{-1}\right)(x)\right) \\ f\left(Q\left(\left(\pi_{1} \circ Q^{-1}\right)(x), \frac{3\left(\pi_{2} \circ Q^{-1}\right)(x)-6(1-t)}{1+2 t}\right)\right) & \text { if } x \in Q\left(\partial M_{1} \times[0,2(1-t)]\right), \\ f(x), & \text { if } x \in Q\left(\partial M_{1} \times[2(1-t), 3]\right), \\ & \text { if } x \in M_{1} \backslash Q\left(\partial M_{1} \times[0,3)\right),\end{cases}
$$

and observe that $H^{3}(\cdot, 0)=H^{2}(\cdot, 1)$ and $H^{3}(\cdot, 1)=f$. Because $K(\cdot, 1), \pi_{1}$, $\left.Q^{-1}\right|_{Q\left(\partial M_{1} \times[0,1)\right)}$ are differentiable mappings, it follows that

$$
\left.H^{2}(\cdot, 0)\right|_{Q\left(\partial M_{1} \times[0,1)\right)}=K(\cdot, 1) \circ \pi_{1} \circ\left(\left.Q^{-1}\right|_{Q\left(\partial M_{1} \times[0,1)\right)}\right)
$$

is differentiable on the open neighbourhood $Q\left(\partial M_{1} \times[0,1)\right)$ of $\partial M_{1}$. Hence, there exists a homotopy $H^{1}: M_{1} \times[0,1] \rightarrow M_{2}$ such that $H^{1}(\cdot, 0)$ is a differentiable mapping, $H^{1}(\cdot, 0) \simeq_{H^{1}} H^{1}(\cdot, 1)\left(\operatorname{rel} \partial M_{1}\right)$ and $H^{1}(\cdot, 1)=H^{2}(\cdot, 0)$ (see [Go, Théorème 4.5 , pp. 64]). Choose $H: M_{1} \times[0,1] \rightarrow M_{2}$ in the following way:

$$
H(x, t)= \begin{cases}H^{3}(x, 1-3 t) & \text { if } 0 \leq t \leq \frac{1}{3} \\ H^{2}(x, 2-3 t) & \text { if } \frac{1}{3} \leq t \leq \frac{2}{3} \\ H^{1}(x, 3-3 t) & \text { if } \frac{2}{3} \leq t \leq 1\end{cases}
$$

Theorem 2.2. Let $M$ be an n-dimensional differentiable manifold $(\partial M=\emptyset)$ and $A$ be a discrete subset of $M$ whose derived set $A^{\prime}$ is also discrete. If $P$ is a compact differentiable $k$-dimensional manifold $(k<n, \partial P \neq \emptyset)$ and $f: P \rightarrow M$ is a continuous mapping such that $f(\partial P) \subseteq M \backslash \bar{A}$, then there exists a continuous mapping $g: P \rightarrow M$ such that $g(P) \subseteq M \backslash \bar{A},\left.g\right|_{\partial P}=\left.f\right|_{\partial P}$ and $f \simeq g(\operatorname{rel} \partial P)$.

Proof. According to Lemma 2.1 there exists a homotopy $H: P \times[0,1] \rightarrow M$ such that $f=H(\cdot, 0), g_{1}=H(\cdot, 1)$ is a differentiable mapping and $H_{t}(\partial P) \subseteq M \backslash \bar{A}$ for all $t \in[0,1]$.

Because $A^{\prime}$ is a closed and discrete subset of $M$ and $g_{1}(P)$ is compact, it follows that $g_{1}(P) \cap A^{\prime}$ is a finite set. Let us assume that $g_{1}(P) \cap A^{\prime}=\left\{x_{1}, x_{2}, \ldots, x_{l}\right\}$. It 
is easily seen that there exist the local charts $c_{1}=\left(U_{1}, \varphi_{1}\right), \ldots, c_{l}=\left(U_{l}, \varphi_{l}\right)$ such that:

(i) $x_{i} \in U_{i}$ and $\varphi_{i}\left(x_{i}\right)=0$ for all $i \in\{1,2, \ldots, l\}$;

(ii) $U_{i} \cap U_{j}=\emptyset$ for $i \neq j$;

(iii) $H_{t}(\partial P) \cap U_{i}=\emptyset$ for all $t \in[0,1]$ and all $i \in\{1, \ldots, l\}$.

Because $A$ and $A^{\prime}$ are discrete sets and $M$ has a countable basis, it follows that both of them are countable, which means that the closure $\bar{A}=A \cup A^{\prime}$ is countable too. We can therefore say that $\varphi_{i}\left(U_{i} \cap \bar{A}\right)$ is countable for each $i \in$ $\{1,2, \ldots, l\}$. But this means that there exists $r>0$ such that $\bar{D}_{r}^{n} \subseteq \bigcap_{i=1}^{l} \varphi_{i}\left(U_{i}\right)$ and $S_{r}^{n-1} \cap \varphi_{i}\left(U_{i} \cap \bar{A}\right)=\emptyset$ for all $i \in\{1,2, \ldots, l\}$. Making, if need be, easy modifications of the mappings $\varphi_{i}$, we can assume that $r=1$. Therefore, $S_{\varphi_{i}} \cap \bar{A}=\emptyset$ or, equivalently, $S_{\varphi_{i}} \subseteq M \backslash \bar{A}$. Therefore the following inclusions

(iv) $\bar{D}^{n} \subseteq \bigcap_{i=1}^{l} \varphi_{i}\left(U_{i}\right)$ and $S_{\varphi_{i}} \subseteq M \backslash \bar{A}$

hold. Obviously $\left[g_{1}(P) \backslash\left(\bigcup_{i=1}^{l} U_{i}\right)\right] \cap \bar{A}$ is a finite set; for instance $\left[g_{1}(P) \backslash\left(\bigcup_{i=1}^{l} U_{i}\right)\right] \cap$ $\bar{A}=\left\{x_{l+1}, \ldots, x_{l+l^{\prime}}\right\}$. Using the same kind of arguments as above, there exist the local charts $c_{l+1}=\left(U_{l+1}, \varphi_{l+1}\right), \ldots, c_{l+l^{\prime}}=\left(U_{l+l^{\prime}}, \varphi_{l+l^{\prime}}\right)$ satisfying the following conditions:

(i') $x_{l+i} \in U_{l+i}$ and $\varphi_{l+i}\left(x_{l+i}\right)=0$ for all $i \in\left\{1, \ldots, l^{\prime}\right\}$;

(ii') $U_{l+i} \cap U_{l+j}=\emptyset$ and $U_{l+i} \cap U_{k}=\emptyset$ for all $i, j \in\left\{1, \ldots, l^{\prime}\right\}, k \in\{1, \ldots, l\}$ and $i \neq j, i \neq k$;

(iii') $H_{t}(\partial P) \cap U_{l+i}=\emptyset$ for all $t \in[0,1]$ and all $i \in\left\{1, \ldots, l^{\prime}\right\}$;

$\left(\mathrm{iv}^{\prime}\right) \bar{D}^{n} \subseteq \bigcap_{i=1}^{l^{\prime}} \varphi_{l+i}\left(U_{l+i}\right)$ and $S_{\varphi_{l+i}} \subseteq M \backslash \bar{A}$ for all $i \in n\left\{1, \ldots, l^{\prime}\right\}$.

Because $g_{1}$ is a differentiable mapping, we obtain, using a weak version of Sard's theorem for the mapping $\left.g_{1}\right|_{\text {int } P}$, that $D_{\varphi_{i}} \backslash g_{1}(P)$ is nonempty for every $i \in\left\{1,2, \ldots, l+l^{\prime}\right\}$. For $i \in\left\{1,2, \ldots, l+l^{\prime}\right\}$, let us consider $y_{i} \in D_{\varphi_{i}} \backslash g_{1}(P)$ and the mappings

$$
\begin{gathered}
g_{c_{i}, y_{i}}: M \backslash\left\{y_{1}, \ldots, y_{l+l^{\prime}}\right\} \rightarrow M \backslash\left\{y_{1}, \ldots, y_{l+l^{\prime}}\right\}, \\
h: P \rightarrow M, h(x)=\left(j \circ g_{c_{1}, y_{1}} \circ \cdots \circ g_{c_{l+l^{\prime}}, y_{l+l^{\prime}}}\right)\left(g_{1}(x)\right)
\end{gathered}
$$

where $j: M \backslash\left\{y_{1}, \ldots, y_{l+l^{\prime}}\right\} \rightarrow M$ is the inclusion map. Obviously, $h(P) \subseteq$ $M \backslash \bar{A},\left.h\right|_{\partial P}=\left.g_{1}\right|_{\partial P}$ and $h \simeq_{G^{\prime}} g_{1}($ rel $\partial P)$, where $G^{\prime}: P \times[0,1] \rightarrow M$ is given by $G^{\prime}(x, t)=G_{c_{1} \cdots c_{l+l^{\prime}}}^{y_{1} \cdots y_{l+l^{\prime}}}\left(g_{1}(x), t\right)$.

By the transitivity of the relation " $\simeq$ ", one can conclude that $f \simeq_{H^{\prime}} h$ where $H^{\prime}: P \times[0,1] \rightarrow M$ is given by

$$
H^{\prime}(x, t)= \begin{cases}H(x, 2 t), & 0 \leq t \leq \frac{1}{2} \\ G^{\prime}(x, 2 t-1), & \frac{1}{2} \leq t \leq 1\end{cases}
$$

Consider the following two homotopies $\psi: P \times[0,1] \rightarrow P$, and $G: P \times[0,1] \rightarrow M$ given by:

$$
\begin{gathered}
\psi(x, t)= \begin{cases}x & \text { if } x \in P \backslash Q(\partial P \times[0,2)), \\
Q\left(\left(\pi_{1} \circ Q^{-1}\right)(x), \frac{2}{2-t}\left(\pi_{2} \circ Q^{-1}\right)(x)+\frac{2 t}{t-2}\right) & \text { if } x \in Q(\partial P \times[t, 2]), \\
\left(\pi_{1} \circ Q^{-1}\right)(x) & \text { if } x \in Q(\partial P \times[0, t]),\end{cases} \\
G(x, t)= \begin{cases}H^{\prime}(\psi(x, t), t) & \text { if } x \in P \backslash Q(\partial P \times[0, t)), \\
H^{\prime}\left(Q^{-1}(x)\right) & \text { if } x \in Q(\partial P \times[0, t]),\end{cases}
\end{gathered}
$$

where $Q: \partial P \times[0, \infty) \rightarrow U \subset P$ is a collar neighbourhood of $\partial P$ and $\pi_{1}: \partial P \times$ $[0, \infty) \rightarrow \partial P, \pi_{2}: \partial P \times[0, \infty) \rightarrow[0, \infty)$ are obviously the projections. 
Denoting $G(\cdot, 1)$ by $g$ and observing that $G(\cdot, 0)=f$ it can be easily seen that $f \simeq_{G} g($ rel $\partial P)$ and also that $g(P) \subseteq M \backslash \bar{A}$, and the theorem is completely proved.

Proposition 2.3. Let $M$ be an $n$-dimensional differentiable manifold $(n \geq 2, \partial M$ $=\emptyset)$ and $A$ be a discrete subset of $M$ whose derived set $A^{\prime}$ is also discrete. If $M$ is connected, then $M \backslash \bar{A}$ is also connected, and the inclusion $i: M \backslash \bar{A} \rightarrow M$ is $(n-1)$-connected; that is, the homomorphism $i_{q}: \pi_{q}(M \backslash \bar{A}) \rightarrow \pi_{q}(M)$, induced by the inclusion, is an isomorphism for $q \leq n-2$ and it is an epimorphism for $q=n-1$.

Proof. The connectedness of $M \backslash \bar{A}$ follows easily from Theorem 2.2, taking the particular case $P=[0,1]$. Using the exact homotopy sequence

$$
\cdots \rightarrow \pi_{i+1}(M, M \backslash \bar{A}) \rightarrow \pi_{i}(M \backslash \bar{A}) \stackrel{i_{*}}{\rightarrow} \pi_{i}(M) \rightarrow \pi_{i}(M, M \backslash \bar{A}) \rightarrow \cdots
$$

it is enough to show that $\pi_{r}(M, M \backslash \bar{A})=0$ for all $r \in\{1,2, \ldots, n-1\}$. But this is an immediate consequence of Theorem 2.2 and of the fact that $[\alpha] \in \pi_{r}(M, M \backslash \bar{A})$ is zero if and only if there exists $\beta \in[\alpha]$ such that $\beta\left(D^{r}\right) \subseteq M \backslash \bar{A}$.

\section{BASIC RESUlT}

Using the results previously obtained, in this section we shall prove the already announced sufficient conditions, in terms of homotopy groups, in order that $\varphi(M, N)$ be infinite, for the given pair $(M, N)$ of differentiable manifolds.

Theorem 3.1. Let $M, N$ be compact connected differentiable manifolds having the same dimension $m$. In these conditions the following statements are true:

(i) If $m \geq 3$ and $\pi_{1}(N)$ has no subgroup isomorphic with $\pi_{1}(M)$, then $\varphi(M, N)=$ $\infty$;

(ii) If $m \geq 4$ and $\pi_{q}(M) \nsucceq \pi_{q}(N)$ for some $q \in\{2,3, \ldots, m-2\}$, then $\varphi(M, N)=$ $\infty$.

Proof. We have to show that any differentiable mapping $f: M \rightarrow N$ has infinitely many critical points. If $f$ is not surjective, this follows easily by Theorem 1.1. Assume that $f: M \rightarrow N$ is surjective and that $f$ has finitely many critical points. Hence, by Theorem 1.2,

$$
g=\left.f\right|_{M \backslash f^{-1}(B(f))}: M \backslash f^{-1}(B(f)) \rightarrow N \backslash B(f)
$$

is a covering map, which means that

$$
g_{1}: \pi_{1}\left(M \backslash f^{-1}(B(f))\right) \rightarrow \pi_{1}(N \backslash B(f))
$$

is a monomorphism and

$$
g_{q}: \pi_{q}\left(M \backslash f^{-1}(B(f))\right) \rightarrow \pi_{q}(N \backslash B(f))
$$

are isomorphisms for all $q \geq 2$. On the other hand, by Theorem 1.2 and Proposition 2.3 it follows that the homomorphisms $i_{q}: \pi_{q}\left(M \backslash f^{-1}(B(f))\right) \rightarrow \pi_{q}(M)$ and $j_{q}$ : $\pi_{q}(N \backslash B(f)) \rightarrow \pi_{q}(N)$ induced by the inclusions $i: M \backslash f^{-1}(B(f)) \rightarrow M$ and $j:$ $N \backslash B(f) \rightarrow N$ are isomorphisms for all $q \in\{0,1, \ldots, m-2\}$. From the commutative 
diagram

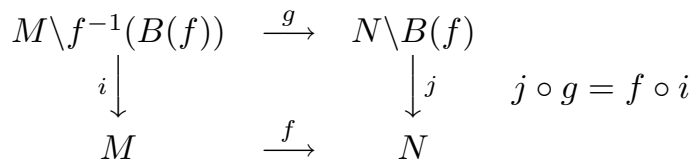

we get the following commutative diagrams:

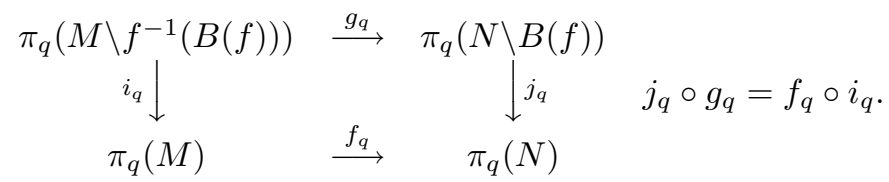

(i) In the case when $q=1$, because $f_{1} \circ i_{1}=j_{1} \circ g_{1}, i_{1}, j_{1}$ are isomorphisms and $g_{1}$ is a monomorphism, it follows that $f_{1}=j_{1} \circ g_{1} \circ i_{1}^{-1}$ is a monomorphism, which is a contradiction with the hypothesis of the statement (i).

(ii) For $q \in\{2, \ldots, m-2\}, i_{q}, j_{q}, g_{q}$ are isomorphisms which together with $f_{q} \circ i_{q}=j_{q} \circ g_{q}$ leads to the conclusion that $f_{q}=j_{q} \circ g_{q} \circ i_{q}^{-1}$ are isomorphisms for all $q \in\{2, \ldots, m-2\}$, which is a contradiction with the hypothesis of the statement (ii).

Corollary 3.2. (i) If $M$ and $N$ are compact diferentiable manifolds having the same dimension $m \geq 3$ and $\left|\pi_{1}(M)\right|>\left|\pi_{1}(N)\right|$, then $\varphi(M, N)=\infty$. So, in particular $\varphi\left(T^{m}, S^{m}\right)=\infty$ and $\varphi\left(P^{n}(\mathbf{R}) \times P^{k}(\mathbf{R}), P^{n+k}(\mathbf{R})\right)=\infty$ where $n, k$ are two natural numbers such that $n+k \geq 3$.

(ii) Under the same conditions on the manifolds $M, N$ as in the statement above and if $\pi_{1}(M)$ and $\pi_{1}(N)$ are finite groups such that $\left(\left|\pi_{1}(M)\right|,\left|\pi_{1}(N)\right|\right)=1$, one gets that $\varphi(M, N)=\infty$.

(iii) If $m, k$ are two natural numbers such that $n, k \geq 2$, then

$$
\begin{aligned}
& \varphi\left(T^{n+k}, T^{n} \times S^{k}\right)=\infty, \varphi\left(T^{n} \times S^{k}, T^{n+k}\right)=\infty, \\
& \varphi\left(S^{n+k}, S^{n} \times S^{k}\right)=\infty, \varphi\left(S^{n} \times S^{k}, S^{n+k}\right)=\infty,
\end{aligned}
$$

where $T^{p}$ denotes the $p$ dimensional torus $\underbrace{S^{1} \times \cdots \times S^{1}}_{p \text { times }}$.

\section{The EQUivariant CASE}

In this section we shall prove an equivariant version of Theorem 3.1.

Let $G$ be a Lie group, $M$ be a manifold and $\varphi: G \times M \rightarrow M,(g, x) \mapsto g x$ be a smooth action of $G$ on $M$. The triple $(G, M, \varphi)$ is called a $G$-manifold. If the action of $G$ on $M$ is free, recall that $M / G$ can be endowed with a differential structure such that the canonical projection $\pi_{M}: M \rightarrow M / G$ is a smooth $G$ bundle. A mapping $f: M \rightarrow N$ between the $G$-manifolds $M$ and $N$ is said to be $G$-equivariant if $f(g x)=g f(x)$ for all $g \in G$ and all $x \in M$. If $M$ and $N$ are two $G$-manifolds and $f: M \rightarrow N$ is smooth $G$-equivariant, denote by $\bar{f}: M / G \rightarrow N / G$ the smooth mapping which makes the following diagram commutative:

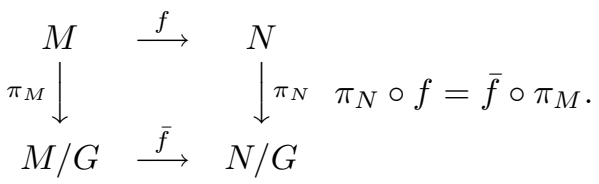


Theorem 4.1. Let $G$ be a connected Lie group and $M, N$ be two compact connected $G$-manifolds such that $\operatorname{dim} M=\operatorname{dim} N$ and the actions of $G$ on $M$ and $N$ are free. Let $k$ be the common dimension of the manifolds $M / G$ and $N / G$. Under these conditions the following assertions are true:

(i) If $k \geq 3, \pi_{1}(M), \pi_{1}(N)$ are commutative groups and $\pi_{1}(N)$ has no subgroup isomorphic with $\pi_{1}(M)$, then any G-equivariant mapping $f: M \rightarrow N$ has infinitely many critical orbits.

(ii) If $k \geq 4$ and $\pi_{q}(M) \neq \pi_{q}(N)$ for some $q \in\{2, \ldots, k-2\}$, then any $G$ equivariant mapping $f: M \rightarrow N$ has infinitely many critical orbits.

Proof. For both of the above assertions it is enough to show that the associated mapping $\bar{f}: M / G \rightarrow N / G$ of an arbitrary $G$-equivariant mapping $f: M \rightarrow N$ has infinitely many critical points. For this purpose, we can assume that $\bar{f}: M / G \rightarrow$ $N / G$ is surjective because otherwise the fact that $\bar{f}$ has infinitely many critical points follows easily from Theorem 1.1. Supposing that $\bar{f}: M / G \rightarrow N / G$ has finitely many critical points, it follows, by Theorem 1.2, that

$$
\bar{g}=\left.\bar{f}\right|_{M / G \backslash \bar{f}^{-1}(B(\bar{f}))}: M / G \backslash \bar{f}^{-1}(B(\bar{f})) \rightarrow N / G \backslash B(\bar{f})
$$

is a covering map, which means that

$$
\bar{g}_{q}: \pi_{q}\left(M / G \backslash \bar{f}^{-1}(B(\bar{f}))\right) \rightarrow \pi_{q}(N / G \backslash B(\bar{f}))
$$

is a monomorphism if $q=1$ and it is an isomorphism if $q \geq 2$. The commutative diagrame

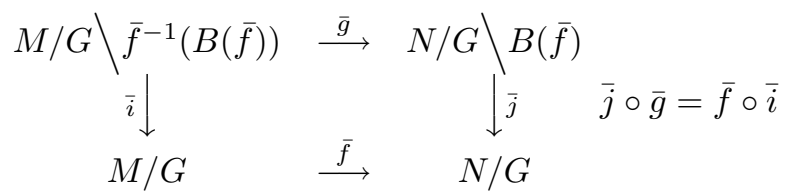

supplies the following commutative diagrams:

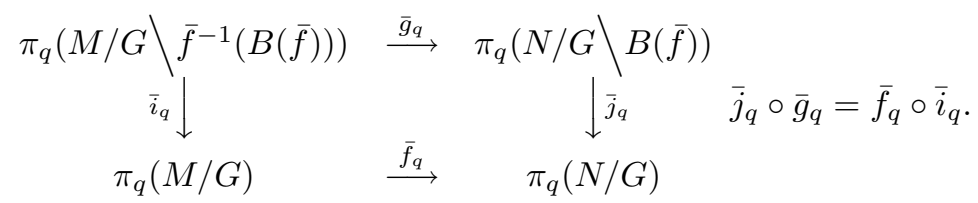

By Proposition 2.3, it follows that for $q \in\{1, \ldots, k-2\}$ the homomorphisms $\bar{i}_{q}$ and $\bar{j}_{q}$ induced by inclusions $\bar{i}$ and $\bar{j}$ are isomorphisms, and they are epimorphisms for $q=k-1$. Therefore $\bar{f}_{q}$ is a monomorphism for $q=1$, it is an isomorphism if $q \in\{2, \ldots, k-2\}$ and $\bar{f}_{k-1}$ is an epimorphism. Consider the following ladder with commutative rectangles:

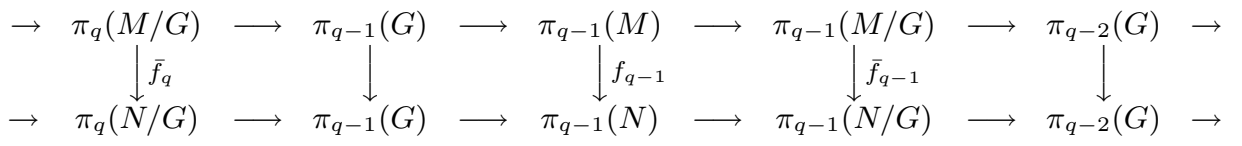

where the rows are the exact homotopy sequences of the principal fibrations $G \hookrightarrow$ $M \rightarrow M / G, G \hookrightarrow N \rightarrow N / G$. Using successively the five lemma for $q=2$ and then for $q \in\{3, \ldots, k-1\}$ in the above diagram, it follows that $f_{1}$ is a monomorphism and $f_{q}$ are isomorphisms for all $q \in\{2, \ldots, k-2\}$, which contradicts the hypothesis of (i) and that of (ii) respectively, and the theorem is completely proved. 
As an application of Theorem 4.1, consider the following free actions of $S^{1}$ on $S^{2 n+1}$ and $T^{2 n+1}$ respectively:

$$
\begin{gathered}
S^{1} \times T^{2 n+1} \rightarrow T^{2 n+1},\left(z,\left(z_{1}, \ldots, z_{2 n+1}\right)\right) \mapsto\left(z^{a_{1}} z_{1}, \ldots, z^{a_{2 n+1}} z_{2 n+1}\right), \\
S^{1} \times S^{2 n+1} \rightarrow S^{2 n+1},\left(z,\left(z_{1}, \ldots, z_{n}\right)\right) \mapsto\left(z z_{1} \ldots, z z_{n}\right),
\end{gathered}
$$

where $n, a_{1}, \ldots, a_{2 n+1}$ are natural numbers such that $n \geq 1$, and $\left(a_{1}, \ldots, a_{2 n+1}\right)=$ 1.

Theorem 4.1 (i) implies that any $S^{1}$-equivariant mapping $f: T^{2 n+1} \rightarrow S^{2 n+1}$ has an infinite number of critical orbits. The fact that any $S^{1}$-equivariant mapping $g: S^{2 n+1} \rightarrow T^{2 n+1}$ has infinitely many critical orbits is proved in the paper [Pi2].

\section{REFERENCES}

[AnPi] D. Andrica, C. Pintea, Critical points of vector-valued functions, Proc. $24^{\text {th }}$ Conf. Geom. Top., Univ. Timişoara.

[Da] R-N. Danuta, Equivariant maps of joins of finite G-sets and an application to critical point theory, Ann. Polonici Math. L VI.2(1992).

[FaHu] E. Fadell, S. Husseini, A note on the category of the free loop space, Proc. Amer. Math. Soc., 107(1989), 527-536. MR 90a:55008

[Go] C. Godbillon, Éléments de Topologie Algébrique, Collection Méthodes, Hermann Paris, 1971. MR 46:880

[GoGo] I.C. Gómez-Larañaga, F. Gonzales-Acuña, Lusternik-Schnirelmann category of 3manifolds, Topology Vol. 31, No. 4, pp. 791-800, 1992. MR 93j:55005

[Pi1] C. Pintea, A measure of non-immersability of the Grassmann manifolds in some Euclidean spaces, Proc. Edinburgh Math. Soc. 41(1998), 197-206. [MR 98k:57051]

[Pi2] C. Pintea, Continuous mappings with an infinite number of topologically critical points, Ann. Polonici Math., LXVII.1 (1997), 87-93. MR 98d:57059

[Ta] F. Takens, The minimal number of critical points of a function on a compact manifold and the Lusternik-Schnirelmann Category, Invent. Math. 6, 197-244(1968). MR 38:5235

Faculty of Mathematics, "Babeģ-Bolyai" University, Str. M. Kogălniceanu 1, 3400 Cluj-Napoca, Romania

E-mail address: cpintea@math.ubbcluj.ro 